\title{
Estabilidade da aptidão física na transição da infância (7-9 anos) para a puberdade (15 anos): o Estudo Morfofuncional da Criança Vianense
}

\author{
Luis Paulo Rodrigues 1,2 \\ Sérgio Angélico 1 \\ Linda Saraiva 1 \\ Pedro Bezerra 1,3
}

https://doi.org/10.5628/rpcd.07.03.347

\author{
1 Instituto Politécnico Viana do Castel o \\ Portugal \\ 2 LIBEC \\ Universidade do M inho \\ Portugal \\ 3 Southern Cross University \\ A ustrália
}

\section{RESUMO}

O objectivo deste estudo foi o de inquirir sobre a estabilidade das trajectórias de aptidão física entre a infância e o final da puberdade. Cento e setenta e quatro rapazes e raparigas foram anualmente avaliados em sete provas constituintes de uma bateria de aptidão física durante três anos consecutivos na infância (7, 8 e 9 anos de idade), sendo sujeitos a uma nova repetição passados seis anos ( 15 anos). A estabilidade dos resultados foi aferida através dos coeficientes de auto-correlação (Spearman e Pearson) e do Kappa de Cohen (K). Os resultados encontrados demonstraram que a estabilidade da aptidão física na transição do período pubertário (9-15 anos) foi menor do que na infância (7-9 anos), e os rapazes foram sempre mais estáveis que as raparigas. Os val ores de auto-correlação interidade variaram de 0,43 a 0,81 entre os 7 e os 9 anos, e de 0,23 a 0,66 dos 9 aos 15 anos. Na generalidade, o tracking encontrado ao longo das idades estudadas foi bastante baixo ( $K$ entre 0,11 a 0,33), mas quando analisada a estabilidade relativa a quatro canais percentílicos, percebe-se que as crianças com piores prestações tendem a permanecer mais no seu canal percentílico ( < p25) enquanto se perspectiva uma maior troca de posições entre os melhores (>p75).

Palavras-chave: tracking, estabilidade, aptidão física, EMCV, Iongitudinal, adolescência.

\begin{abstract}
Tracking physical fitness from childhood (7-to-9 years old) to late puberty (15 years-old): the Estudo M orfofuncional da Criança Vianense.

O ur main goal in this study was to understand the stability and tracking of physical fitness from childhood to late puberty. O ne hundred seventy four Portuguese boys and girls, participants on the Estudo M orfofuncional da Criança Vianense, were annually assessed on seven physical fitness tests for three consecutive times during prepubertal years (ages 7-, 8-, and 9-years old), and once at late puberty (15-years old).

Tracking was assessed using auto-correlation coefficients (Spearman and Pearson) and Cohen's Kappa (K). Results showed higher stability during pre-puberty years (7-to-9-years old) compared with pubertal years ( 9 -to-15-years-old), with boys' fitness levels being more stable than girls. 0 verall ageto-age correlations for 7 to 9 years old ranged between 0,43 and 0,81 , and 0,23 and 0,66 for 5 to 15 years old. Tracking across all ages was low ( $\mathrm{K}$ ranging from 0,11 to 0,33 ), but children classified in the lower quartile $(<$ p25) tended more to remain on the same performance channel, while the ones classified in the higher quartile (> p75) changed position more often
\end{abstract}

Key-words: tracking, physical fitness, EM CV, longitudinal, adolescence. 


\section{INTRODUÇÃO}

A crescente preocupação com a implementação de estilos de vida activos leva a que os níveis de aptidão física (ApF) das populações jovens sejam encarados hoje em dia como factor fundamental na potenciação das melhorias de saúde. As evidências actuais apontam para um declínio crescente dos níveis de aptidão física na criança e no jovem(24, 26, 33). Ao mesmo tempo, verifica-se uma redução acentuada dos tempos e intensidades de actividade física e um aumento dos factores de risco para a saúde em populações cada vez mais jovens, o que sugere a importância da aptidão física na infância como factor protector deste fenómeno(8,15,16,17). Aliás, na aproximação, já clássica, da relação entre aptidão física e actividade física, estas são consideradas como factores que se influenciam mutuamente na determinação da saúde na adolescência e idade adulta(21).

Apesar de existir um vasto conhecimento nacional e internacional acerca da aptidão física das crianças e jovens, os estudos longitudinais que nos podem permitir inferir acerca das reais trajectórias de desenvolvimento são reconhecidamente escassos e centramse essencialmente no período pós-pubertário e na passagem para a idade adulta( $(5,6)$. Neste contexto, 0 estudo da estabilidade (ou tracking), entendida como a manutenção das posições relativas de uma variável no seio do grupo de pertença, assume uma relevância fundamental para a mel hor compreensão das trajectórias individuais da ApF ao longo do crescimento e na sua relação particular com a saúde(17).

O que sabemos hoje sobre a aptidão física de crianças sugere a existência de uma grande variabilidade interindividual, que tende a aumentar com idade. Presumir que a ApF se desenvolve de forma estável pressupõe um risco aumentado para as crianças que exibem desde cedo menor proficiência, já que não só continuariam nessa situação ao longo do crescimento como ainda por cima tenderiam a registar menos melhorias. Ilustrativo deste exemplo são os resultados da prova de resistência aeróbia incluída no Estudo Morfofuncional da Criança Vianense, onde os valores entre os seis e os dez anos do p90 melhoraram cerca de três vezes mais que os do $\mathrm{p} 10 \mathrm{em}$ ambos os $\operatorname{sexos}(28,29)$.

O conhecimento do fenómeno da maior ou menor estabilidade da ApF é ainda incipiente no que diz respeito à passagem da infância para o final da puberdade. A maior parte dos estudos tem-se sobretudo debruçado sobre as relações entre o final da puberdade e a idade adulta, com resultados indicativos de tracking moderado a alto nas diversas componentes da $\operatorname{ApF}(25,5,21)$. $O$ facto de os valores registados para a estabilidade da ApF na idade adulta serem quase sempre mais elevados do que os relativos à actividade física(25, 21) reforça a utilidade do tracking na ApF durante a infância e juventude. A existência de razoáveis níveis de estabilidade entre a infância e pós-puberdade seria pois motivo para reforçar a validade do diagnóstico e estimulação precoce da ApF como estratégia de intervenção num momento em que as crianças se encontram especialmente sensíveis às modificações comportamentais. Deste modo poder-se-ão assegurar melhores níveis de aptidão física na passagem para a vida adulta, com a consequente adopção de estilos de vida mais saudáveis. Este cenário parece ser suportado por al guns resultados que sugerem que os mais aptos durante a infância tendem a perservar esta característica durante a adolescência(17) e a ser mais activos na idade adul ta(21, 11).

Entre as várias componentes da aptidão física, a resistência aeróbia, a força abdominal e a flexibilidade têm sido apontadas (conjuntamente com a composição corporal que não é tratada neste estudo) como as mais relacionadas com a saúde geral (health-related physical fitness). A aptidão aeróbia parece apresentar uma estabilidade moderada durante o período correspondente à passagem da infância para a puberdade, com os rapazes a evidenciarem geralmente val ores mais elevados que os pares do sexo oposto. Os valores de auto-correlações encontrados na literatura referem-se a provas diferenciadas $\left(\mathrm{VO}_{2 \text { máx }}\right.$, corrida de 600 metros, corrida da milha) e idades iniciais também distintas mas com intervalos de tempo semelhantes ( $\approx 4$ anos). São referidas correlações de 0,44 e 0,39 entre as idades de 10-14 anos, 0,62 e 0,40 para os 7-11 anos, e 0,56 e 0,42 aos 9-12 anos, respectivamente para rapazes e raparigas, e de 0,55 para jovens entre os oito e os doze anos de idade(14, 17, 23, 31). A estabilidade das prestações de força relatadas na literatura varia segundo os grupos musculares testados. $\mathrm{Na}$ força superior, medida pelo tempo máximo de suspensão na barra, os val ores situam-se entre 
0,52 e 0,54 para rapazes ( 8 aos 14, e 10 aos 16 anos), e 0,44 em raparigas entre os oito e os catorze $\operatorname{anos}^{(9,12)}$. Nas prestações de força média abdominal (entre os 10-16 e 9-12 anos) foram encontradas correlações de 0,40 e 0,46 para os elementos do sexo masculino e de 0,47 no sexo feminino(12). Na força inferior e no período entre os sete e os onze anos de idade, rapazes e raparigas obtiveram valores respectivamente de 0,43 e 0,40 na prova de salto em comprimento sem corrida preparatória(14).

A estabilidade da velocidade tem sido pouco estudada neste período etário, mas numa prova de velocidade lançada de 15 e 20 metros a auto-correlação encontrada entre os sete e os onze anos de idade foi de 0,54 nos rapazes e 0,50 para as raparigas(14). $\mathrm{Na}$ flexibilidade têm sido encontrados valores de estabilidade razoáveis no teste de sit-and-reach, variando entre 0,52 e 0,67 nos rapazes, e 0,44 e 0,72 nas raparigas, entre os 8-14 e 9-12 anos de idade respectivamente ${ }^{(9,23)}$.

No presente estudo, e dada a fal ta de estudos de natureza longitudinal relatando este comportamento, propusemo-nos inquirir sobre a estabilidade das trajectórias de ApF entre a infância e o final da puberdade. É nossa expectativa poder ajudar a perceber melhor a natureza do desenvolvimento das componentes da ApF numa fase julgada tão importante da vida as crianças e jovens. Será que as crianças mais aptas crescem naturalmente para serem jovens igualmente aptos ou a "norma" (e o inverso com os menos aptos)? Qual é a força do condicionamento, a influência, que os níveis de ApF exibidos em criança exercem sobre os momentos mais tardios de vida? Será que a puberdade se perfila como momento singular na determinação das trajectórias de ApF? Será que a estabilidade (ou falta dela) neste período de vida é semelhante para todas as componentes de ApF e independente do género?

Estas são al gumas das questões que pretendemos abordar nesta investigação, em que 174 rapazes e raparigas foram anual mente avaliados em sete provas constituintes de uma bateria de ApF durante três anos consecutivos na infância ( 7, 8 e 9 anos de idade), sendo sujeitos a uma nova repetição passados seis anos (15 anos). À luz dos resultados encontrados esperamos poder melhor perspectivar as intervenções na melhoria da ApF na infância e juventude.

\section{MATERIAL E MÉTODOS}

$O$ delineamento experimental deste estudo é tipicamente longitudinal apesar de não apresentar uniformidade nos interval os de testagem. A amostra definida foi avaliada em quatro momentos distintos, três deles antes da puberdade ( 7,8 , e 9 anos) e um situado num momento tardio ou mesmo final do salto pubertário ( 15 anos). O salto de seis anos na recolha de dados teve como propósito explícito evitar as perturbações naturais decorrentes da grande variabilidade dos ritmos individuais característicos do período pubertário, e retomar a análise num momento final ou próximo do final deste período. Não foram utilizados neste estudo quaisquer indicadores do estatuto maturacional das crianças e jovens que nos permitam assegurar que este intervalo de seis anos incluiu o momento de sal to pubertário para todas os sujeitos, mas crianças europeias e norte-americanas têm sido geralmente classificadas no primeiro estádio dos indicadores sexuais secundários aos 9 anos (pré-puberdade), e no último estádio, indicativo do final da puberdade, aos 15 anos(22).

Os dados relativos aos três primeiros momentos foram extraídos do EMCV, investigação que decorreu de 1997 a 2000 e que recolheu dados morfológicos, bio-sociais e de aptidão física de 2386 crianças pertencentes a quinze escolas do 1o Cido do Ensino Básico de Viana do Castelo. Assim, vamos referir-nos apenas aos procedimentos relativos ao quarto momento de recolha de dados (15 anos), já que os três primeiros foram já descritos anteriormente $(28,29,30)$.

\section{Amostra}

Este estudo apresenta os resultados das componentes de ApF de 174 jovens ( 101 rapazes e 73 raparigas) que foram avaliados longitudinalmente em quatro momentos, correspondendo a uma média de idade similar para ambos os géneros, de respectivamente 7,1, 8,1, 9,1 e 15,1 anos ( $\mathrm{DP}=0,4)$. A amostra foi desenhada com o objectivo de seguir após a puberdade as crianças pertencentes ao EMCV. De um conjunto inicial de 435 elementos (203 rapazes, 216 raparigas), foram identificados 325 jovens em nove escolas EB2,3 e Secundárias do Concelho de Viana do Castelo (EB 2,3 Frei Bartolomeu dos Mártires; EB 2,3 Viana do Castelo; EB 2,3 Dr. Pedro 
Barbosa; EB 2,3 Pintor José de Brito; EB 2,3 de Lanheses; Externato das Neves; ES Santa Maria Maior; ES Monserrate). Desta amostra, três alunos desistiram de estudar e outros três não foram autorizados pelos pais a participar no estudo, 13 encontravam-se doentes ou lesionados no momento da avaliação, 23 recusaram-se a realizar os testes de ApF, e não foi possível avaliar outros 37 alunos devido a ausência dos docentes ou dos próprios alunos no dia marcado para a avaliação dos testes de ApF.

Consequentemente foram avaliados 246 al unos, representando $76 \%$ dos al unos que haviam sido previamente identificados e que permaneceram no sistema educativo no concel ho. Destes, e dada a natureza longitudinal desta análise, são apresentados neste estudo apenas os dados referentes aos 174 jovens que participaram em todos os quatro momentos de testagem.

Testes de Aptidão Física

A bateria de $\mathrm{ApF}$ utilizada foi a previamente adoptada no EMCV(29) e realizou-se na seguinte ordem: teste de tempo máximo de suspensão na barra (TSB), sit-and-reach (SR), corrida de agilidade $4 \times 10$ metros (shuttlerun) (SHR), número de abdominais em 60 segundos com membros inferiores flectidos e membros superiores cruzados sobre o peito (ABD), salto em comprimento sem corrida preparatória (SCP), a corrida de velocidade em 50 metros (C50), e a corrida de resistência em vai vém de 20 metros (CVV).

Recolha de dados

Foram solicitadas e obtidas autorizações do Centro de Área Educativa de Viana do Castelo, dos Consel hos Executivos e dos Conselhos Pedgaógicos das Escolas, dos professores de Educação Física das turmas envolvidas, e dos pais e dos jovens pertencentes à amostra. No caso dos pedidos de autorização entregues aos alunos/ pais eram incluídos os valores anteriormente registados pelo aluno (e só dele) nos quatro anos do EMCV. Esta estratégia foi utilizada para aumentar o interesse dos al unos na participação nos testes. A pós o período de testagens, todas as escolas e al unos envolvidos receberam uma informação contendo os seus resultados individuais e médios da escola.
Devido à diversidade de escolas e turmas envolvidas, a equipa de observadores foi constituída por seis elementos que, em grupos de dois, se deslocaram a cada escola para realizar a avaliação de ApF nas aulas de Educação Física e na presença do professor da turma. A testagem foi inteiramente conduzida nas instalações (ginásios e espaços desportivos exteriores) utilizadas pelas escolas envolvidas e decorreu durante os meses de Abril e Maio. No total foram visitadas 89 turmas, contendo cada uma de 1 a 21 al unos a serem testados. Todos os procedimentos utilizados respeitaram as normas internacionais de experimentação com humanos, expressas na Declaração de Helsínquia de 1975.

Controlo de qualidade dos dados

A execução dos testes obedeceu aos protocolos descritos nas baterias de teste de onde são originários( $(1,2,10)$. Na C50 e SHR foram registados dois tempos, sendo a média considerada como resultado final. As equipas de observação, constituídas por jovens licenciados do Curso de Educação Física, foram previamente treinadas nas tarefas específicas que desempenhavam. De acordo com os procedimentos anteriormente utilizados no EMCV, um em cada doze alunos foi escolhido al eatoriamente para repetir a execução dos testes (à excepção dos ABD e CVV, devido ao elevado esforço dispendido para a sua realização), com a finalidade de se aferir a fiabilidade da avaliação. Os coeficientes de correlação intra-classe resultantes desta repetição situam-se entre os 0,78 no TSB e os 0,95 no SR, dentro dos limites considerados como normais para um estudo desta natureza(32).

Os dados finais, após introdução na base de dados informatizada, foram escrutinados para detecção de possíveis erros. O registo de distribuição de cada variável foi analisado e todos os valores detectados como extremos foram reconfirmados nos registos originais e corrigidos ou apagados (nos casos em que existia erro evidente no registo original).

Procedimentos estatísticos

O comportamento de cada variável ao longo das diferentes idades e segundo o sexo, é descrito através dos valores da média (M) \pm desvio-padrão (DP). A normalidade da distribuiçao das variáveis em cada 
Quadro 1. Valores descritivos (número, média e desvio padrão) dos testes de aptidão física em rapazes e raparigas ao longo dos quatro momentos de observação.

\begin{tabular}{lcccccc}
\hline & & & 7 anos & 8 anos & 9 anos & 15 anos \\
\hline Prova & Sexo & $n$ & M DP & M DP & M \pm DP & M DP \\
\hline ABD & Masc & 98 & $20,5 \pm 7,8$ & $25,2 \pm 7,8$ & $30,7 \pm 7,5$ & $43,5 \pm 9,2$ \\
& Fem & 72 & $19,9 \pm 7,2$ & $23,1 \pm 8,0$ & $27,8 \pm 8,8$ & $35,1 \pm 11,3$ \\
\hline TSB & Masc & 77 & $14,5 \pm 11,6$ & $11,7 \pm 11,0$ & $13,2 \pm 9,9$ & $34,2 \pm 14,9$ \\
& Fem & 70 & $14,4 \pm 12,6$ & $12,7 \pm 11,8$ & $14,7 \pm 12,3$ & $19,3 \pm 12,6$ \\
\hline SCP & Masc & 97 & $107,5 \pm 18,2$ & $113,4 \pm 18,8$ & $118,6 \pm 18,7$ & $189,1 \pm 28,8$ \\
& Fem & 72 & $100,5 \pm 16,4$ & $105,0 \pm 15,4$ & $108,9 \pm 16,4$ & $156,2 \pm 22,3$ \\
\hline C50 & Masc & 94 & $11,4 \pm 1,1$ & $10,8 \pm 1,0$ & $10,2 \pm 1,0$ & $7,8 \pm 0,9$ \\
& Fem & 68 & $12,2 \pm 1,2$ & $11,3 \pm 1,0$ & $10,8 \pm 0,9$ & $8,9 \pm 1,1$ \\
\hline SHR & Masc & 98 & $13,1 \pm 1,0$ & $12,4 \pm 0,9$ & $12,2 \pm 1,0$ & $10,8 \pm 1,2$ \\
& Fem & 72 & $13,8 \pm 1,1$ & $13,0 \pm 1,1$ & $12,6 \pm 0,8$ & $11,8 \pm 1,1$ \\
\hline CW & Masc & 89 & $24,8 \pm 12,0$ & $33,3 \pm 15,0$ & $37,5 \pm 16,1$ & $58,7 \pm 21,5$ \\
& Fem & 67 & $21,8 \pm 10,6$ & $25,6 \pm 10,8$ & $28,8 \pm 12,3$ & $31,2 \pm 12,9$ \\
\hline SR & Masc & 101 & $25,6 \pm 5,8$ & $25,0 \pm 6,3$ & $23,6 \pm 6,3$ & $24,6 \pm 7,6$ \\
& Fem & 73 & $27,8 \pm 5,0$ & $27,3 \pm 6,0$ & $26,6 \pm 6,1$ & $30,0 \pm 5,9$ \\
\hline
\end{tabular}

Masc = masculino; Fem = feminino. TSB - teste de tempo máximo de suspensão na barra; SR - sit-and-reach; SHR corrida de agilidade 4x10 metros (shuttle-run); ABD - número de abdominais em 60 segundos; SCP - salto em comprimento sem corrida preparatória; C50 - corrida de velocidade em 50 metros; CW corrida de resistência em vaivém de 20 metros

momento foi escrutinada através do teste de Shapiro Wilks. O coeficiente de auto-correlação de Spearman foi utilizado para descrever a estabilidade das prestações momento-a-momento nos casos em que se detectaram distribuições não normais ( $A B D, T S B$, C50, SHR, CVV, eSR). No teste de SH foi empregue o coeficiente de auto-correlação de Pearson. Como val ores de referência entende-se que auto-correlações abaixo de 0,30 são indicadoras de baixa estabilidade idade a idade, entre 0,30 e 0,60 estabilidade moderada, e acima deste valor a estabilidade é considerada de moderada a al ta(20).

Para avaliar a estabilidade dos resultados ao longo dos quatro momentos (tracking) foi utilizado o Kappa de Cohen $(K)$ que testa a permanência dos sujeitos no mesmo canal ao longo do tempo. Foi previamente estipulada a divisão em quatro canais percentílicos com os valores de corte relativos a p25, p50 e p75. Este procedimento estatístico não-paramétrico resiste a violações dos pressupostos de normalidade das distribuições, e corrige para a utilização de intervalos desiguais de recol ha de dados tal como acontece no nosso delineamento experimental. Segundo as propostas de Landis e Koch(19) um valor de K $>0.75$ indica estabilidade excelente, $K$ entre 0,40 e 0,75 estabilidade moderada a boa, e valores abaixo de 0.40 demonstram estabilidade baixa.

A estatística descritiva e as auto-correlações idade a idade foram obtidas no programa SPSS 11.0. Os valores de tracking foram encontrados recorrendo ao programa estatístico Longitudinal D ata A nalisys (LDA).

\section{RESULTADOS}

Resultados dos valores médios

No Quadro 1 são apresentados os valores médios e respectivos desvios-padrão dos testes de ApF para cada momento de testagem e por sexo.

Durante os quatro momentos de testagem verificaram-se melhorias gerais no desempenho médio de todas as componentes da ApF, excepção feita ao teste 
Quadro 2. Coeficientes de auto-correlação (idade-a-idade) das prestações nos testes de aptidão física de rapazes e raparigas.

ABD

\begin{tabular}{l|cccc}
\multicolumn{1}{c}{} & 7 anos & 8 anos & 9 anos & 15 anos \\
\cline { 2 - 5 } 7 anos & - & 0,54 & 0,46 & 0,43 \\
8 anos & 0,61 & - & 0,60 & 0,37 \\
9 anos & 0,49 & 0,51 & - & 0,38 \\
15 anos & 0,38 & 0,43 & 0,23 & -
\end{tabular}

$\mathrm{SCP}$

7 anos

8 anos

9 anos

15 anos

\begin{tabular}{|cccc}
\multicolumn{5}{c}{7 anos } & 8 anos & 9 anos & 15 anos \\
\hline- & 0,73 & 0,70 & 0,53 \\
0,76 & - & 0,72 & 0,61 \\
0,68 & 0,85 & - & 0,69 \\
0,48 & 0,52 & 0,59 & -
\end{tabular}

SHR

7 anos

8 anos

9 anos

15 anos

\begin{tabular}{|cccc}
\multicolumn{5}{c}{7 anos } & 8 anos & 9 anos & 15 anos \\
\hline- & 0,76 & 0,66 & 0,34 \\
0,75 & - & 0,74 & 0,39 \\
0,73 & 0,70 & - & 0,45 \\
0,34 & 0,25 & 0,44 & -
\end{tabular}

SR

7 anos

8 anos

9 anos

15 anos

\begin{tabular}{|cccc}
\multicolumn{5}{c}{ T anos } & 8 anos & 9 anos & 15 anos \\
\hline 0,78 & 0,68 & 0,64 & 0,45 \\
0,78 & 0,81 & 0,77 & 0,64 \\
0,53 & 0,63 & 0,66 & 0,63 \\
\hline
\end{tabular}

TSB

\begin{tabular}{ccccc}
7 anos & 8 anos & 9 anos & 15 anos & \\
\hline- & 0,76 & 0,65 & 0,44 & 7 anos \\
0,65 & - & 0,80 & 0,44 & 8 anos \\
0,66 & 0,72 & - & 0,42 & 9 anos \\
0,42 & 0,42 & 0,52 & - & 15 anos
\end{tabular}

C50

\begin{tabular}{ccccc}
7 anos & 8 anos & 9 anos & 15 anos & \\
\hline- & 0,71 & 0,63 & 0,42 & 7 anos \\
0,74 & - & 0,74 & 0,58 & 8 anos \\
0,70 & 0,697 & - & 0,48 & 9 anos \\
0,45 & 0,360 & 0,56 & - & 15 anos
\end{tabular}

\begin{tabular}{ccccc}
\multicolumn{7}{c}{ CW } \\
7 anos & 8 anos & 9 anos & 15 anos & \\
\cline { 1 - 4 }- & 0,60 & 0,43 & 0,22 & 7 anos \\
0,62 & - & 0,46 & 0,31 & 8 anos \\
0,55 & 0,73 & - & 0,33 & 9 anos \\
0,49 & 0,52 & 0,63 & - & 15 anos
\end{tabular}

Nota: todos os coeficientes são significativos ( $p<01$ ) com excepção dos encontrados na CW feminino aos 15-8 anos ( $p<.05$ ), e 15-7 anos (não significativo). TSB - teste de tempo máximo de suspensão na barra; SR - sit-and-reach; SHR corrida de agilidade $4 \times 10$ metros (shuttle-run); ABD - número de abdominais em 60 segundos; SCP - salto em comprimento sem corrida preparatória; C50 - corrida de velocidade em 50 metros; CW corrida de resistência em vaivém de 20 metros

de flexibilidade do tronco (SR). Neste último, e como geralmente tem sido descrito(2, 18), existe uma pequena diminuição ao longo do período pré-pubertário ( $7,8,9$ anos) para depois se verificar uma melhoria, em particular nas raparigas, durante 0 salto pubertário.

Os rapazes obtiveram melhores prestações médias em seis dos sete testes ( ABD, SCP, TSB, C50, SHR e CVV), sendo as raparigas mais proficientes no $S R$. Demorando o nosso olhar especificamente nas transformações ocorridas durante o período pubertário (9 aos 15 anos) reparamos que as melhorias demons- tradas pelos rapazes são muito maiores do que as das raparigas, fenómeno naturalmente associado ao dimorfismo sexual no crescimento morfológico ocorrido neste período. De notar que as maiores evidências destas diferenças se encontram na aptidão aeróbia (CVV) com um incremento médio de 21 percursos nos rapazes versus apenas 2 nas raparigas, e na força superior (TSB) (21 versus 5 segundos).

Resultados de estabilidade / tracking Os resultados das autocorrelações entre os diversos momentos para cada um dos testes de ApF estão 
Quadro 3. Valores de tracking (Kappa de Cohen geral e respectivos intervalos de confiança e Kappa por canal quartílico) para cada um dos testes de aptidão física para os rapazes e raparigas.

\begin{tabular}{llllcccc}
\hline & & Kappa & $95 \%$ l & & $\begin{array}{c}\text { kappa } \\
\text { p25-p50 }\end{array}$ & $\begin{array}{c}\text { track } \\
\text { p50-p75 }\end{array}$ & $>$ >p75 \\
\hline ABD & Fem & 0,22 & $0,16-0,28$ & 0,32 & 0,16 & 0,06 & 0,32 \\
& Mas & 0,19 & $0,14-0,23$ & 0,29 & 0,06 & 0,10 & 0,32 \\
\hline TSB & Fem & 0,23 & $0,18-0,29$ & 0,31 & 0,06 & 0,13 & 0,43 \\
& Mas & 0,26 & $0,20-0,31$ & 0,46 & 0,12 & 0,11 & 0,33 \\
\hline SCP & Fem & 0,29 & $0,24-0,35$ & 0,46 & 0,11 & 0,16 & 0,43 \\
& Mas & 0,33 & $0,29-0,38$ & 0,51 & 0,25 & 0,17 & 0,39 \\
\hline C50 & Fem & 0,21 & $0,16-0,27$ & 0,33 & 0,13 & 0,05 & 0,36 \\
& Mas & 0,24 & $0,19-0,29$ & 0,43 & 0,13 & 0,03 & 0,38 \\
\hline SHR & Fem & 0,24 & $0,18-0,30$ & 0,32 & 0,08 & 0,20 & 0,36 \\
& Mas & 0,27 & $0,22-0,32$ & 0,42 & 0,13 & 0,11 & 0,44 \\
\hline CW & Fem & 0,11 & $0,05-0,16$ & 0,21 & 0,00 & $-0,01$ & 0,23 \\
& Mas & 0,23 & $0,18-0,28$ & 0,41 & 0,04 & 0,11 & 0,34 \\
\hline SR & Fem & 0,26 & $0,21-0,32$ & 0,50 & 0,11 & 0,05 & 0,37 \\
& Mas & 0,33 & $0,28-0,37$ & 0,52 & 0,13 & 0,20 & 0,44 \\
\hline
\end{tabular}

Masc = masculino; Fem = feminino. TSB - teste de tempo máximo de suspensão na barra; SR - sit-and-reach; SHR corrida de agilidade 4x10 metros (shuttle-run); ABD - número de abdominais em 60 segundos; SCP - salto em comprimento sem corrida preparatória; C50 - corrida de velocidade em 50 metros; CW corrida de resistência em vaivém de 20 metros

representados no Quadro 2. Os valores encontrados para o sexo masculino figuram na metade inferior esquerda e os do sexo feminino na metade superior direita da diagonal.

Relativamente ao período pré-pubertário (7-9 anos), os valores de auto-correlação demonstram a existência de uma estabilidade razoável no desempenho dos testes de TSB, SH, C50, SHR e SR em ambos os sexos, e na CVV somente para os rapazes. Quando reparamos no padrão das correlações neste mesmo período verificamos que a estabilidade da prestação parece aumentar com a idade nos testes ABD e C50m nas raparigas, nos SH e CVV nos rapazes, e nos TSB e SR para ambos os sexos.

Analisando os valores das auto-correlações entre os dois momentos considerados como imediatamente antes e após a puberdade ( 9 el5 anos) encontramos val ores de estabilidade moderada $(0,52$ a 0,66$)$ para as prestações dos rapazes no TSB, SH, C50, CVV e SR, e apenas no SH e SR para as raparigas, sendo todos os outros desempenhos considerados como pouco estáveis nesta transição da idade pubertária. Reparando no padrão mais global das matrizes de auto-correl ação - que nos dá uma ideia mais global da estabilidade do desempenho ao longo de todos os momentos testados - constatamos mais uma vez que apenas o SH, CVV, e o SR nos rapazes; e o SH e o SR nas raparigas, revelam valores de estabilidade moderada no desempenho longitudinal. Neste tipo de análise global destacam-se também, mas pelos baixos val ores de estabilidade exibidos, as prestações na CVV das raparigas. Esta particularidade é aliás a única característica distintiva entre os sexos no que ao padrão geral das auto-correlações diz respeito, já que em todas as outras provas o comportamento é bastante idêntico.

O valor do Kappa de Coehen ( $K$ ) permitenos estabelecer a estabilidade da trajectória dos sujeitos ao longo de vários momentos no tempo, testando a sua permanência no mesmo canal percentílico de desen- 
volvimento. O facto de esta análise utilizar a informação de todos os momentos de testagem proporciona uma medida mais ajustada à verdadei ra noção de traço da aptidão física do indivíduo (estabilidade das posições relativas dos sujeitos ao longo do tempo). Para além da medida geral, este teste estatístico permite-nos ter uma visão do comportamento dos sujeitos em cada um dos canais escolhidos, permitindo na prática diferenciar traçados de estabilidade segundo a maior ou menor proficiência exibida. No Quadro 3 podemos encontrar os valores do $K$ geral e respectivos interval os de confiança ( $95 \%$ IC) para cada um dos testes de ApF segundo o sexo. Estão ainda representados os $\mathrm{K}$ parciais para cada um dos quatro canais percentílicos de performance previamente escolhidos e que nos permitem perceber o seu tracking específico.

$\mathrm{Na}$ generalidade o tracking encontrado para cada um dos testes de $A p F$ é bastante baixa ( $K$ entre 0,11 a $0,33)$, indiciando falta de estabilidade no desempenho de rapazes e raparigas em todas as provas e ao longo das idades estudadas. Destacam-se com os valores mais baixos os rapazes na prova de $A B D$ $(0,19)$ e as raparigas na de CVV $(0,11)$. A pesar de os elementos do sexo masculino apresentarem quase sempre valores de $K$ mais elevados (com excepção da prova de $A B D$ ), estas diferenças não são suficientes para inferir de qualquer significado estatístico na comparação da estabilidade entre sexos (valores não mostrados).

Quando analisamos a indicação da estabilidade relativa aos quatro canais percentílicos estabelecidos é possível constatar uma realidade um pouco diferente. Como é usual, o tracking encontrado nos canais de desempenho centrais ( $p 25-p 50-p 75$ ) é muito baixo, sendo mais elevado entre os que se encontram nos dois extremos: os mais fracos e os mais proficientes. Ainda assim, e no canal indicador das melhores performances (>p75) apenas nas provas de TSB e SH das raparigas, e nas de SHR e SR dos rapazes se verificaram indícios moderados de estabilidade $(0,43$ a 0,44). Já no primeiro canal (o de piores desempenhos) foram encontrados valores indicativos de estabilidade moderada $(0,41$ a 0,52$)$ em seis dos sete testes nos rapazes (TSB, SH, C50, SHR, CVV, e $\mathrm{SR}$ ), mas apenas em dois nas raparigas ( $\mathrm{SH}$ e SR, respectivamente 0,46 e 0,50).

\section{DISCUSSÃO}

Não existem na literatura muitos estudos de nature za longitudinal relatando a auto-correlação das componentes de aptidão física em idades pré-pubertárias e na passagem para a puberdade. Além disso os poucos estudos existentes variam imenso no tipo de provas efectuadas, no tempo que medeia entre as observações, no intervalo etário consagrado e consequentemente nos ritmos de maturação biológica, e no ambiente envolvente, o que dificulta a comparação de valores. Parece, no entanto, haver concordância entre os autores de que a estabilidade destas componentes da ApF é ligeiramente mai or durante o período pré-pubertário do que entre este e o final da puberdade $(9,14,23)$. No resumo dos estudos existentes (Quadro 4), os autores referem a existência de valores de estabilidade moderada (de 0,40 a 0,62) ao longo de um período de quatro anos na infância (610 anos e 7-11 anos), decrescendo ligeiramente $(0,39$ a 0,54$)$ quando o período de recolha passou a incluir o salto pubertário.

Nos nossos dados, os valores de auto-correlação entre os 7 e os 9 anos variaram de 0,43 a 0,81, revelando na generalidade uma estabilidade moderada (ABD, CVV), a moderada alta (TSB, SH, C50, SHR, $\mathrm{SR})$, com os valores mais elevados a pertencerem ao teste de flexibilidade do tronco $(0,68-0,81)$.

Os valores mais baixos de estabilidade registaram-se nas provas de abdominais em ambos os sexos $(0,46$ a 0,61 ) e de CVV no sexo feminino $(0,43$ a 0,60$)$ constituindo esta última uma observação comum a outros autores (14, 23, 17, 31). A menor familiaridade das crianças com este tipo de provas poderá ajudar a explicar estes valores, traduzindo-se numa dificuldade coordenativa na execução do movimento de força abdominal ou, no caso da CVV, do esforço para completar a prova.

Também pelo que ficou anteriormente dito seria de esperar as auto-correl ações nas crianças e jovens aqui descritos pudessem ser maiores entre os 9-7 anos do que entre os 15-9 anos, o que de facto aconteceu na generalidade das provas. No entanto, os valores da auto-correlação das provas de SCP e SR mantiveram-se acima (ou muito próximo) do valor considerado como de estabilidade moderada alta $(0,60)$ nos dois momentos, sendo mesmo que nas raparigas apenas decresceram 0.01 de um momento 
Quadro 4. Valores de correlações idade-a-idade das componentes de aptidão física de rapazes e raparigas em vários estudos.

\begin{tabular}{|c|c|c|c|c|}
\hline & Intervalo etário & Masc & Fem & \\
\hline \multicolumn{5}{|l|}{ Aptidão Aeróbica } \\
\hline \multirow[t]{4}{*}{ VO2 max (ml. $\left.\mathrm{min}^{-1} \cdot \mathrm{kg}^{-1}\right)$} & $6-10$ & & 0,49 & Saris et al (1986) \\
\hline & $8-12$ & & 0,55 & \\
\hline & $6-12$ & & 0,56 & \\
\hline & $10.8-14.6$ & 0,44 & 0,39 & Janz et al (2000) \\
\hline $600 \mathrm{~m}$ & $7-11$ & 0,62 & 0,42 & Falk et al (2001) \\
\hline Milha & $9-12$ & 0,56 & 0,42 & Marshall et al (1998) \\
\hline \multicolumn{5}{|l|}{ Velocidade } \\
\hline $15 / 20$ m sprint & $7-11$ & 0,54 & 0,50 & Falk et al (2001) \\
\hline \multicolumn{5}{|l|}{ Força } \\
\hline SCP & $7-11$ & 0,43 & 0,40 & Falk et al (2001) \\
\hline \multirow[t]{2}{*}{$A B D$} & $10-16$ & 0,40 & & Ellis et al (1975) \\
\hline & $9-12$ & 0,46 & 0,47 & Marshall et al (1998) \\
\hline \multirow[t]{2}{*}{ TSB } & $10-16$ & 0,54 & & Ellis et al (1975) \\
\hline & $8-14$ & 0,52 & 0,44 & Branta et al (1984) \\
\hline \multicolumn{5}{|l|}{ Flexibilidade } \\
\hline \multirow[t]{2}{*}{ SR } & $9-12$ & 0,67 & 0,72 & Marshall et al (1998) \\
\hline & $8-14$ & 0,52 & 0,44 & Branta et al (1984) \\
\hline
\end{tabular}

Masc = masculino; Fem = feminino. TSB - teste de tempo máximo de suspensão na barra; SR - sit-and-reach; ABD - número de abdominais em 60 segundos; SCP - salto em comprimento sem corrida preparatória.

para o outro $(0,70$ para 0,69 , e 0,64 para 0,63 , respectivamente no SCP e SR), o que parece indicar mesmo (e dado o maior intervalo de tempo) um ganho de estabilidade neste segundo momento. Para além disso, e ao contrário de todas as outras provas, os rapazes melhoraram a estabilidade da sua prestação na CVV no intervalo dos 9 aos 15 anos. Esta melhoria poderá dever-se à mai or dificuldade sentida pelas crianças mais novas para cumprirem os requisitos da prova (apesar de termos tentado minimizar estas dificuldades tendo sempre um adulto a correr com as crianças, marcando o ritmo da prova e incentivando à sua execução máxima), ou pela especial ização metabólica que tem sido descrito ocorrer durante a puberdade(4) e que resulta num perfil mais aeróbio ou anaeróbico do jovem, e logo numa maior estabilidade do seu desempenho.

A análise da estabilidade ao longo de todo o período considerado ( 7 aos 15 anos) através do Kappa de Cohen e segundo os níveis de prestação (canais quartílicos) demonstra que, embora com valores de expectativa moderada, as crianças com piores prestações tendem a permanecer mais no seu canal percentílico ( $<$ p25) enquanto se perspectiva uma maior troca de posições entre os melhores ( $>p 75)$. Estes resultados, aliados aos baixos valores gerais de estabilidade em todas as provas de ApF, parecem indicar que prestações passadas não asseguram necessariamente resultados futuros no comportamento destas variáveis, mas também, com tudo o que esta conclusão tem de gravosos para a perpetuação de baixos níveis de ApF nos percursos de desenvolvimento individual das crianças e jovens, que as lacunas sentidas pelas crianças menos aptas parecem ser as mais difíceis de corrigir.

A interpretação estrita desta falta generalizada de estabilidade nas trajectórias da infância para a puberdade tardia pode ter várias leituras. Por um lado parece ser característica humana (a que os investigadores dificilmente conseguem escapar) a esperança 
de encontrar comportamentos previsíveis, logo estáveis, ao longo do desenvolvimento. Esta assumpção traduz-se na interpretação quase sempre abonatória da existência de traços estáveis, trazendo assim alguma segurança à nossa compreensão do fenómeno e à consequente avaliação e diagnóstico preditivo das situações de excepcional idade futuras ( risco e rendimento). Por outro lado esta mesma interpretação traz consigo um carácter determinista e uma consequência de quase inevitabilidade a que nem sempre temos prestado a devida atenção. De facto, altos níveis de estabilidade nas prestações de ApF durante um período tão alargado e repleto de mudanças como é o estudado ( 7 aos 15 anos), constituiria uma prova da inevitabilidade do nosso destino genético, capaz de resistir a tantas influências ambientais a que as nossas crianças e jovens estão naturalmente sujeitos. Não seria por isso, estamos certos, uma boa notícia para todos os que se dedicam à promoção da ApF com a convicção de que a estimulação é elemento fulcral nesta matéria.

Em síntese, poderemos concluir que a estabilidade da ApF na transição do período pubertário (9-15 anos) é menor do que na infância ( $7-9$ anos) em qualquer dos sexos, mas os rapazes apresentam níveis de ApF mais estáveis do que as raparigas, quer observando os períodos separadamente quer quando entramos com todos os momentos na análise longitudinal. Entre as provas realizadas, a força média abdominal (ABD) para ambos os sexos, e a resistência aeróbia (CVV) nas raparigas, apresentam-se com os mais baixos índices de estabilidade. Esta conclusão é sobremaneira importante já que, sendo estes itens normalmente associados à saúde dos indivíduos, a sua pouca estabilidade coloca dificuldades na detecção precoce de problemas.

Demonstrada a baixa estabilidade longitudinal das componentes da ApF, ressalta das conclusões deste estudo a possibilidade clara das modificações das trajectórias dos indivíduos com relevância especial nas mudanças para níveis mais elevados. Uma palavra de cautela deve ser deixada no que diz respeito às crianças colocadas muito cedo nos níveis mais baixos e que parecem, essas sim, ainda mais necessitadas de intervenção precoce dada a sua maior aderência a este estado de menor proficiência.

\section{AGRADECIMENTOS}

Este estudo foi parcialmente suportado por uma bolsa do Programa PAFID, Projecto Ref №18/2006, do Instituto de Desporto de Portugal .

Os autores querem ainda expressar os seus mais profundos agradecimentos à Câmara Municipal de Viana do Castelo pelo apoio prestado, às escolas e respectivos professores e alunos pela sua colaboração desinteressada, e aos elementos da equipa de observação do quarto momento (Drs Arlindo Peixoto, Raul Oliveira, João Rocha, João Sá, e Daniel Rafael) pela sua colaboração inestimável.

\section{CORRESPONDÊNCIA}

\section{Luís Paulo Rodrigues}

Escola Superior Educação Viana do Castelo

Av. Capitão Gaspar de Castro

A partado 513

4901-908 Viana do Castelo

Telefone 258806200

Telefax 258806209

E-mail: Iprodrigues@ese.ipvc.pt 
BIBLIOGRAFIA

1. AAHPERD (1976). Youth Fitness Test M anual. Washington, DC.

2. AAHPERD (1980). H ealth Related Physical Fitness M anual. Washington, DC.

3. Baquet G, Twisk J, Kemper H, Van Praagh E, Berthoin S (2006). Longitudinal follow-up of fitness during childhood: interaction with physical activity. A m J H um Biol, 18(1): 51-58.

4. Bar-Or O (1983). Pediatric Sports M edicine. Berlin: SpringerVerlag.

5. Beunen G, Ostyn M, Simons J, Renson R, Claessens AL, Vanden Eynde B, Lefevre J, Vanreusel B, Malina RM, van't Hof MA (1997). Development and tracking in fitness components: Leuven longtudinal study on lifestyle, fitness and health. Int J Sports M ed 18 Suppl 3:S171-178.

6. Beunen GP, Philippaerts RM, Delvaux K, Thomis M, Claessens AL, Vanreusel B, Eynde BV, Lysens R, Renson R, Lefevre J (2001) Adolescent physical performance and adult physical activity in Flemish males. A m J Hum Biol 13:173-179.

7. Boreham C, Robson P, Gallagher A, Cran G, Savage JM, Murray $L$ (2004). Tracking of physical activity, fitness, body composition and diet from adolescence to young adulthood: The Young Hearts Project, Northern Ireland. Int J Behav Nutr Phys Act 1(1): 14.

8. Boreham C; Riddoch C (2001). The physical activity, fitness and health of children. J Sports Sci 19 (12): 915-929.

9. Branta C, Haubenstricker J, Seefeldt V (1984) Age changes in motor skills during childhood and adolescence. Exerc Sports Sci Rev 12: 467-520.

10. Comitato Olimpico Nazionale Italiano(1988). EUROFIT $\mathrm{H}$ andbook. Rome.

11. Dennison B, Straus A, Mellits E, Charney E (1988). Childhood physical fitness tests: Predictor of adult physical activity levels? Pediatrics 82: 324-330.

12. Ellis D, Carron AV, Bailey DA (1975). Physical performance in boys from 10 through 16 years. $\mathrm{Hum} \mathrm{Biol} \mathrm{47:} \mathrm{263-}$ 281.

13. Falk B, Bar-Or B (1993). Longitudinal changes in peak aerobic and anaerobic mechanical power of circumpubertal boys. Ped Exerc Sci 5: 318-331

14. Falk B, Cohen Y, Lustig G, Lander Y, Yaaron M, Ayalon J (2001). Tracking of physical fitness components in boys and girls from the second to sixth grades. A m J H um Biol 13(1), 65-70

15. Hofman A, Walter H J (1989). The association between physical fitness and cardiovascular disease risk factors in children in a five-year follow-up study. I J Epidemiol 18: 830-835.

16. Janz K F , Mahoney L T (1997). Three-year follow-up of changes in aerobic fitness during puberty: the muscatine study. Res Q Exerc Sport 68 (1): 1-10.

17. Janz K F, Dawson J D, L. T. Mahoney L T (2000). Tracking physical fitness and physical activity from childhood to adolescence: the Muscatine study. M ed Sci Sports Exerc 32: 1250-1257.

18. Kemper H, Nels J, Verschuur R, Storm-van Essen L (1990). Tracking of health and risk indicators of cardiovascular diseases from teenager to adulthood: Amsterdam Growth and Health Study. Prev M ed 19: 642-655.
19. Landis J, Koch $G$ (1977). The measurement of observer agreement for categorical data, Biometrics 33: 159-174.

20. Malina R M (1996). Tracking of physical activity and physical fitness across the lifespan. Res Q Exerc Sport 67: 48-57.

21. Malina R M (2001). Physical activity and fitness: Pathways from childhood to adulthood. A m J H um Biol 13(2): 162172.

22. Malina R, Bouchard C (1991). Growth, maturation and physical activity. Champaign: Human Kinetics)

23. Marshall SJ, Sarkin JA, Sallis JF, McKenzie TL (1998). Tracking of health-related fitness components in youth ages 9 to 12. M ed Sci Sports Exerc 30: 910-916.

24. Matton L, Duvigneaud N, Wijndaele K, Philippaerts R, Duquet W, Beunen G, Claessens, Thomis M, Lefevre J (2007). Secular trends in anthropometric chatacteristics, physical fitness, physical activity, and biological maturation in Flemish adolescents between 1969 and 2005. A m J H um Biol 19: 345-357.

25. Matton L, Thomis M, Wijndaele K, Duvigneaud N, Beunen G; Claessens A, Vanreusel B, Philippaerts R, Lefevre J (2006). Tracking of Physical Fitness and Physical Activity from Youth to Adulthood in Females. M ed Sci Sports Exerc 38(6): 1114-1120.

26. Olds T, Tomkinson G, Léger L, Cazorla G (2006). Worldwide variation in the performance of children and adolescents: an analysis of 109 studies of the 20-m shuttle run test in 37 countries. J Sports Sci 24(10): 1025-1038.

27. Pate R R, Dowda M, Ross J G (1990). Associations between physical activity and physical fitness in American Children. A m J Dis Child 144: 1123-1129.

28. Rodrigues L, Bezerra P, Saraiva L (2005). Influência do meio (urbano e rural) no padrão de aptidão física de rapazes de Viana do Castelo, Portugal. R evista Portuguesa Ciências D esporto 5(1): 77-84.

29. Rodrigues L, Sá C., Bezerra P, Saraiva L (2006). Estudo M orfofuncional da Criança Vianense. CMVC: Viana do Castelo.

30. Rodrigues L, Saraiva L, Bezerra P (in press). Aptidão Física no 1ำ Ciclo do Ensino Básico em Viana do Castelo. H orizonte.

31. Saris W, Elvers J, van't Hof M, Binkhorst R (1986). Changes in physical activity of children aged 6 to 12 years. In J Rutenfraz, R ocellin, F Klimt (Eds), Children and exercise XII, (pp 121-130). Champaign, IL: Human Kinetics

32. Shrout, P, Fleiss, J (1979). Intraclass correlations: Uses in assessing rater reliability. Psychol Bull 86(2): 420-428.

33. Sugihara T, Kondo M, Mori S, Yoshida I (2006). Chronological change in preschool children's motor ability development in Japan from the 1960 s to the 2000s. Int J Sport Health Sci, 4: 49-56. 